\title{
Clinical manifestation, laboratory findings, and outcome of children with cystic fibrosis over a 10-year period in South Iran
}

\author{
Fateme Ziyaee', Seyed Mohsen Dehghani ${ }^{2}$, Sima Hosseini', Mohammad Ebrahim Zohalinezhad 3,4, \\ Soheyla Aleyasin ${ }^{6}$ and Hazhir Javaherizadeh ${ }^{7,8^{*}}$ (i)
}

\begin{abstract}
Background: Cystic fibrosis (CF) is a monogenic hereditary disease with diverse complications, which substantially reduce the quality of life and longevity of patients. With regard to the scarcity of information on epidemiological aspects of this disease in Iran, we aimed to examine the current status of pediatric CF patients in a tertiary referral center in south Iran.

Results: Medical records of 105 patients (51\% female) with the mean age of $16.3 \pm 34.4$ months were reviewed. Twelve patients (13.3\%) had siblings with CF. Failure to thrive and respiratory symptoms were the most common presentations. Elevated liver enzymes and steatorrhea were detected in over half of tested patients, while abnormal serum albumin level, urinalysis, positive urine cultures, and positive acute phase reactants were noticed in less than half of the patients. Mild increase in pulmonary artery blood pressure was the most common finding of echocardiography. Increased liver echogenicity was the top finding of abdominopelvic sonography. Out of 42 available patients, 19 (45.2\%) died with the mean age of $54.4 \pm 101.8$ months. The highest rate of mortality was observed in the 2-6years old age group (42.9\%).

Conclusion: Increased liver echogenicity was the most common finding in hepatobiliary evaluation. Mild increase in pulmonary artery blood pressure was the most common finding of echocardiography.
\end{abstract}

Keywords: Cystic fibrosis, Demography, Epidemiology, Genetic diseases, Inborn, Pediatrics

\section{Background}

Cystic fibrosis (CF) is the most common lethal autosomal recessive genetic disorder in white ethnicity with a global estimated prevalence of 80,000 [1]. CF was first described in 1938 as a distinct entity [2], and its responsible gene, CF Transmembrane Conductance Regulator (CFTR) gene, was identified half a century later on chromosome 7 [3, 4]. CFTR functions as an ATP ion channel that regulates the transport of chloride at the

\footnotetext{
* Correspondence: Hazhirja@yahoo.com

${ }^{7}$ Alimentary Tract Research Center, Ahvaz Jundishapur University of Medical Sciences, Ahvaz, Iran

${ }^{8}$ Department of Pediatric Gastroenterology, Abuzar Children's Hospital, Ahvaz 61636-14175, Iran

Full list of author information is available at the end of the article
}

apical membrane of epithelial cells [5]. Nearly 2000 detected mutations in the encoding gene of CFTR result in variable degree of dysfunction to absence of this protein [6]. Consequently, decreased mucociliary clearance (MCC) and accumulation of dehydrated thickened secretions occurs in organs with epithelialized surfaces, such as airways, pancreatic ducts, hepatobiliary ducts, gastrointestinal tract (GIT), and genitourinary tract. Based on the severity of the genetic defect, these conditions are associated with a wide range of CF-related comorbidities in patients [7], including but not limited to obstructive lung disease, endobronchial infections, pancreatic insufficiency, malnutrition, and failure to thrive $[8,9]$. Therefore, management of CF is multidisciplinary and mainly targeted at the specific complications of patients, for 
instance pancreatic enzymes replacement and nutritional support, antibiotic therapy, administration of antiinflammatory agents, mucolytics, and more recently novel CFTR modulators [10-13]. These strategies have successfully improved the survival and life-expectancy over the past decades [14].

Although CF is relatively rare in Iran, the great personal and financial burden of the disease and the availability of effective treatment modalities justify the efforts on expanding our understandings about CF epidemiology and regional differences. This will help diagnose the disease at earlier stages and minimize the chance of neglecting new cases, particularly in pediatric field, where prompt and aggressive initiation of treatment can highly influence the outcome. Following this concern and due to the lack of information on epidemiology of CF in our province, we conducted this study to examine the current status of pediatric CF patients in a tertiary referral center in south Iran.

\section{Methods}

\section{Patients and settings}

This retrospective cross-sectional study was conducted on CF patients (< 18 years), who had been diagnosed by pediatric gastroenterologists or pulmonologists. Medical records of patients were extracted from the registry of our university clinic in Iran, over a 10 -year period from 2002 to 2011. Diagnosis was confirmed if along with related clinical manifestations and/or positive family history of CF, two separate samplings revealed sweat chloride concentration to be above $60 \mathrm{mmol} / \mathrm{lit}$. Patients were excluded if CF was ruled out in further follow-ups.

\section{Data gathering}

Demographic characteristics and medical profile including age at the time of diagnosis, gender, initial manifestation of disease, and family history were recorded. Laboratory and paraclinical findings such as complete blood count (CBD), serum level of acute phase reactants including erythrocyte sedimentation rate (ESR) and Creactive protein (CRP), liver enzymes (AST and ALT), albumin, blood urea nitrogen, creatinine, blood culture, urinalysis and urine culture, stool exam for occult blood, occult parasite and fat, blood gases, plasma electrolytes, as well as echocardiography and sonography results were assessed. Outcome of cases was collected.

Data were analyzed using Windows SPSS software, version 22 , and a $p$ value less than 0.05 was considered as the statistical level of significance.

\section{Results}

A total of 105 medical records were reviewed, of whom $51 \%$ were female and $49 \%$ were male. The mean age of patients was $16.28 \pm 34.36$ months (10 days to 204 months). Twelve patients (13.3\%) had siblings with CF. We divided the patients into age-groups with cutoffs at 6 months, 2 years, and 6 years for better evaluation.

\section{Clinical manifestations}

Failure to thrive and respiratory symptoms such as cough were the most common presentations. As seen in Table 1, abdominal pain was the most frequent in children aged $>6$ years compared to the other age group.

\section{Laboratory findings and imaging}

The results of laboratory tests are demonstrated in Table 2. There were no significant differences among age groups.

Liver function test was available in the documents of 67 patients, most of whom had abnormally high levels of (> $40 \mathrm{U} / \mathrm{L}$ ) ALT (74.6\%) and AST (58.2\%), and among $36 \%$ of them, serum albumin level were less than $3 \mathrm{~g} / \mathrm{L}$.

Out of 75 urinalysis results, $30(40 \%)$ were found abnormal due to glycosuria $(n=13)$, hematuria $(n=8)$, pyuria $(n=8)$, and proteinuria $(n=4)$. Positive urine cultures were detected in $15(23.4 \%)$ of 64 tested patients.

Among 84 patients, for whom blood gases analysis was requested, alkalosis was noticed in 35 (41.7\%), while only 13(15.7\%) had acidosis. Acute phase reactants, including ESR and CRP were elevated in $23(37.7 \%)$ out of 61 and 19 (38.8\%) out of 49 patients, respectively (Table 2). The overall incidence of positive blood cultures and bacteremia was $10(50 \%)$ out of 20 patients. Fatty stool was detected in $45(84.9 \%)$ of 53 , occult blood in 9 (21.4\%) of 42, and parasite in 1 of 39 specimens.

Echocardiography was performed for 25 patients. Various abnormalities were detected in $80 \%$ of these patients as follows: mild increase in pulmonary artery blood pressure (60\%), mild to moderate tricuspid regurgitation (50\%), atrial septal defect (25\%), pulmonary valve insufficiency (15\%), patent ductus arteriosus (15\%), and patent foramen ovale (15\%).

In addition, 15 out of 38 patients who underwent abdominopelvic sonography showed the following abnormalities: increased liver echogenicity $(n=8)$, hepatomegaly $(n=4)$, splenomegaly $(n=1)$, severe ascites $(n=1)$, ectopic left kidney $(n=1)$, gallbladder thickening $(n=1)$, intestinal wall thickening $(n=1)$, hyper-echogenicity of right kidney $(n=1)$, and multiple hypo-echogenic lesions in liver $(n=1)$.

\section{Medical therapy}

Medical therapy was the main treatment approach. Of all cases, 90\% had received antibiotics, and cephalosporins were most commonly administered (71.4\%). Pancreatic enzymes (73\%), supplements (87.6\%), bronchodilators (28.4\%), and mucolytics 
Table 1 The frequency of respiratory, gastrointestinal, and systemic presentations in our study population of CF patients categorized by age groups

\begin{tabular}{|c|c|c|c|c|c|c|}
\hline Presentation & $<6$ months & 6 months to 2 years & $2-6$ years & 6 years $<$ & Total & $P$ value \\
\hline \multicolumn{7}{|l|}{ Respiratory } \\
\hline Cough & $54.8 \%$ & $51.7 \%$ & $42.9 \%$ & $57.1 \%$ & $56(53.3 \%)$ & 0.936 \\
\hline Increased respiratory rate & $38.7 \%$ & $34.5 \%$ & $42.9 \%$ & $42.9 \%$ & $40(38.1 \%)$ & 0.985 \\
\hline Respiratory distress & $37.1 \%$ & $41.4 \% \%$ & $42.9 \%$ & $42.9 \%$ & $41(39 \%)$ & 0.983 \\
\hline Sputum & $27.9 \%$ & $37.9 \%$ & $42.9 \%$ & $42.9 \%$ & $34(32.4 \%)$ & 0.648 \\
\hline \multicolumn{7}{|l|}{ Gastrointestinal } \\
\hline Abdominal pain & 0 & 0 & $14.3 \%$ & $28.6 \% \%$ & $3(2.9 \%)$ & $0.002^{*}$ \\
\hline Flatulence & $9.7 \%$ & $3.4 \%$ & $28.6 \%$ & 0 & $9(8.6 \%)$ & 0.114 \\
\hline Nausea/vomiting & $37.1 \%$ & $37.9 \%$ & $14.3 \%$ & $14.3 \%$ & $36(34.3 \%)$ & 0.429 \\
\hline Distal bowel obstruction & $3.2 \%$ & 0 & $14.3 \%$ & 0 & $3(2.9 \%)$ & 0.234 \\
\hline Delayed meconium passage & $12.9 \%$ & 0 & 0 & 0 & $8(7.6 \%)$ & 0.084 \\
\hline Diarrhea & $33.3 \%$ & $37.9 \%$ & $28.6 \%$ & $28.6 \%$ & 36 (34.3\%) & 0.848 \\
\hline Constipation & 0 & $6.9 \%$ & 0 & $14.3 \%$ & $3(2.9 \%)$ & 0.096 \\
\hline \multicolumn{7}{|l|}{ Systemic } \\
\hline Failure to thrive & $56.5 \%$ & $55.2 \%$ & $28.6 \%$ & $42.9 \%$ & $56(53.3 \%)$ & 0.542 \\
\hline Fever & $24.2 \%$ & $55.2 \%$ & $28.6 \%$ & $42.9 \%$ & $36(34.3 \%)$ & $0.029^{*}$ \\
\hline Convulsion & $1.6 \%$ & 0 & $14.3 \%$ & 0 & $2(1.9 \%)$ & 0.249 \\
\hline Irritability & $16.1 \%$ & $20.7 \%$ & $14.3 \%$ & 0 & 17 (16.2\%) & 0.672 \\
\hline
\end{tabular}

(6.3\%) were also prescribed for the treatment protocol.

\section{Patients' outcome}

Forty-two patients could be contacted for outcome assessment. Nineteen (45.23\%) had died with the mean age

Table 2 Laboratory findings in our study population of CF patients

\begin{tabular}{llll}
\hline Parameters & Minimum & Maximum & Mean \pm SD \\
\hline WBC & 1000 & 26,000 & $13100 \pm 4980$ \\
Hemoglobin & 4 & 18 & $10.86 \pm 3.08$ \\
MCV $^{a}$ & 55 & 113 & $79.08 \pm 15.54$ \\
MCH $^{b}$ & 23 & 36.00 & $25.05 \pm 4.84$ \\
PIt $\times 10^{3}$ & 9.8 & 984 & $387.2 \pm 207.5$ \\
AST & 10 & 417 & $91.4 \pm 73.23$ \\
ALT & 4 & 421 & $54.69 \pm 65.99$ \\
Albumin & 1.3 & 5.4 & $3.49 \pm 0.91$ \\
Total bilirubin & 0.2 & 17 & $1.83 \pm 3.35$ \\
Direct bilirubin & 0.1 & 10.50 & $0.66 \pm 1.46$ \\
BUN & 1 & 76 & $11.95 \pm 11.28$ \\
Creatinine & 0.1 & 2.5 & $0.6 \pm 0.27$ \\
ESR & 2 & 68 & $19.83 \pm 18.04$ \\
Sweat Cl test & 60 & 198 & $92.85 \pm 41.60$ \\
\hline aMean corpuscular volume & & \\
bMean corpuscular hemoglobin & &
\end{tabular}

of $54.4 \pm 101.8$ months. The highest rate of mortality was observed in the 2-6years old age group (42.9\%). The mean age of alive patients was $74.6 \pm 43.8$ months. Concurrent respiratory and gastrointestinal diseases were reported in 13 patients, while 9 had only gastrointestinal complications (Table 3).

\section{Discussion}

The number of patients with CF is relatively limited in Iran. Our center, as a large tertiary referral hospital, evidently provides care and services for all severe cases of pediatric CF. The pediatric outpatient clinic is linked to the hospital, where attending professors visit and refer suspected patients for admission and further work-ups. Over 10 years, 105 CF patients had been admitted. In our study population, the ratio of male to female was 1:1.

On average, patients were diagnosed in the second year of life. In another study, $64 \%$ of the children were diagnosed $<3$ years of age [15].

Alkalosis was more prevalent in arterial blood gas analysis compared to acidosis. In a systematic review by Scurati-Manzoni et al., metabolic alkalosis was similar between subacute and chronic patient with cystic fibrosis [16].

Acute phase reactants were in slightly over one third of tested patients positive. It may be due to recurrent pulmonary infection in children with CF. 
Table 3 Complications and outcome in our study population of CF patients

\begin{tabular}{lllll}
\hline Outcome & $<\mathbf{6}$ months & $\mathbf{6}$ months to $\mathbf{2}$ years & $\mathbf{2 - 6}$ years & $\mathbf{6}$ years < \\
\hline Healthy & $1.6 \%$ & $3.4 \%$ & 0 & 0 \\
Gl complications & $8.1 \%$ & $13.8 \%$ & 0 & 0 \\
Respiratory complications & 0 & $10.3 \%$ & 0 & $14.3 \%$ \\
Concurrent Gl and respiratory complications & $17.7 \%$ & $6.9 \%$ & $42.9 \%$ & $28.6 \%$ \\
Dead & $22.6 \%$ & $65.5 \%$ & $42.9 \%$ & $57.1 \%$ \\
Unavailable & $50 \%$ & & \\
\hline
\end{tabular}

Most common findings in echocardiography were mild increase in pulmonary artery blood pressure and mild to moderate tricuspid regurgitation. Increased pulmonary artery blood pressure is the early sign of potential heart impairment in pediatric age group with CF [17].

Increased liver echogenicity was seen in $21 \%$ of children who underwent abdominal sonography. In the study by Akata et al., increased liver echogenicity was seen in 50\% of children with CF [18]. This difference may be due to disease stage and also expertise of radiologist.

Severe anemia was seen in our study. Early severe anemia may be the first sign of cystic fibrosis [19].

In the current study, $13.3 \%$ of the cases had sibling with CF. Positive consanguinity was reported in $50 \%$ of patients in another study from Egypt [20].

Several population-specific studies in Iran have detected novel mutations and polymorphisms in CFTR gene [21-25]. In the Mohseni et al.'s study, novel compound heterozygous missense mutation (c.3119 T>A (p.L1040H)), which was previously reported as nonsense c.3484C > T (p.R1162X) mutation, was found in exon 19 in patient screening [21]. In the study by Alibakhshi et al., the most common mutations were p.F508del (DeltaF508) (18.1\%), c.2183_2184delAAinsG (2183AA>G) (6.5\%), p.S466X (5.8\%), p.N1303K (4.3\%), c.2789+5G>A (4.3\%), p.G542X (3.6\%), c.3120+1G>A (3.6\%), p.R334W (2.9\%), and c.3130delA (2.9\%) [22]. Distribution of mutations are not consistent with other populations, which emphasizes the necessity of adopting modified sequence analysis for appropriate screening and genetic counseling programs in Iranian CF patients [26-28]. Furthermore, the clinical role of these variations should be considered as phenotype is related to the genotype [29].

Similar to our study, respiratory symptoms such as cough and pulmonary infection, steatorrhea, FTT [30], diarrhea [30], respiratory distress [31-33], alkalosis, and liver function abnormalities [30] have been reported to be frequent among Iranian $\mathrm{CF}$ patients. In a study from Egypt, chronic cough, failure to thrive, and steatorrhea were the most common findings [20]. FTT was reported in all children in a study from Egypt [34].
Mortality rate was $45.23 \%$ among children with follow-up. In the study by Smith et al., among children with CF who admitted to pediatric intensive care unit, mortality rate was $19.1 \%$ which is significantly lower than our study [35].

Significant associations between serum albumin and protein with weight, height, and head circumference have been suggested [36]. In a study by Vahedi et al., it was stated that positive family history, a consanguineous marriage, and residence in a rural area increases the rate of mortality [37]. In addition, pseudomonas infection, number of previous admissions and the severity of pulmonary hypertension were mentioned as major determinants of mortality by Baghaie et al. [38].

The scarcity of CF studies in low prevalence countries such as Iran in contrast to the extent of European and American literature is not surprising. However, it would be a mistake to ignore the importance of fatal rare diseases, when effective managements can be applied. Failing to raise awareness in medical community leads to underdiagnosis or misdiagnosis, especially in a disease like CF which has diverse presentations. The population of Iran is heterogeneous and comprises of multiple ethnicities. Therefore, regional studies are encouraged to verify the spectrum of CF manifestations, investigate unknown causes of neonatal mortality more precisely, and conduct genetic testing to reveal specific CFTR mutations and their consequent outcomes and predicted survival.

It is well-know that earlier presentation is associated with severity of CF, while patients diagnosed in adulthood have milder clinical phenotypes. Thus, our findings cannot accurately represent the general status of all CF patients. Due to the limited number of patients, researcher could benefit most from national registries [39].

\section{Conclusion}

Increased liver echogenicity was the most common finding in hepatobiliary evaluation. Mild increase in pulmonary artery blood pressure was the most common finding of echocardiography. Establishing a single national database yields more reliable information and allows for prospective following of mortality trends, addressing the 
impact of attributable factors, monitoring the efficacy and pitfalls of care, and prioritizing required interventions in different centers.

\section{Limitation}

Cross-sectional study and single-center study are the limitations of the current study. Genetic study was not available for most of the patients due to economic problem.

\section{Abbreviations}

ALT: Alanine amino transferase; AST: Aspartate amino transferase; BUN: Blood urea nitrogen; CF: Cystic fibrosis; CFTR: Cystic fibrosis transmembrane conductor regulator; CRP: C-Reactive protein; ESR: Erythrocyte sedimentation rate

\section{Acknowledgements}

This study was supported by research affair of Shiraz University of Medical Sciences.

\section{Authors' contributions}

FZ collected the data and wrote draft of proposal and performed data analysis. SMD had main idea and wrote the manuscript and also has a role in the revision of the manuscript. SH has a role in data collection, data analysis, and revision of the manuscript. MEZ has a role in writing the manuscript and revision of the manuscript. SA supervised the thesis and collected data and also participated in main idea. $\mathrm{HJ}$ performed the literature review and has a role in writing manuscript, revision of manuscript, and data analysis. All the authors read and approved the manuscript.

\section{Funding}

No funding

\section{Availability of data and materials} Yes

\section{Ethics approval and consent to participate}

This study was approved by ethical committee of Shiraz University of Medical Sciences. The protocol of study was in accordance with the Helsinki Declaration of bioethics and approved by institutional review board. Confidentiality of patients' information was ensured. Verbal consent was obtained from patients/lawful guardians. This study was registered by number 3227 at research affair of Shiraz University of Medical Sciences.

\section{Consent for publication}

Nothing to declare

\section{Competing interests}

Nothing to declare

\footnotetext{
Author details

'Department of Pediatrics, Shiraz University of Medical Sciences, Shiraz, Iran. ${ }^{2}$ Gastroenterohepatology Research Center, Shiraz University of Medical Sciences, Shiraz, Iran. ${ }^{3}$ Department of Traditional Persian Medicine, Shiraz University of Medical Sciences, Shiraz, Iran. ${ }^{4}$ Research Center for Traditional Medicine and History of Medicine, Shiraz University of Medical Sciences, Shiraz, Iran. ${ }^{5}$ Essence of Parsiyan Wisdom Institute, Traditional Medicine and Medicinal Plant Incubator, Shiraz University of Medical Sciences, Shiraz, Iran. ${ }^{6}$ Department of Pediatric Immunology and Allergy, Nemazee Teaching Hospital, Shiraz University of Medical Sciences, Shiraz, Iran. ${ }^{7}$ Alimentary Trac Research Center, Ahvaz Jundishapur University of Medical Sciences, Ahvaz, Iran. ${ }^{8}$ Department of Pediatric Gastroenterology, Abuzar Children's Hospital, Ahvaz 61636-14175, Iran
}

Received: 24 March 2020 Accepted: 25 October 2020

Published online: 23 November 2020

\section{References}

1. Cohen-Cymberknoh M, Shoseyov D, Kerem E (2011) Managing cystic fibrosis: strategies that increase life expectancy and improve quality of life. Am J Respir Crit Care Med 183(11):1463-1471

2. Andersen $\mathrm{DH}$ (1938) Cystic fibrosis of the pancreas and its relation to celiac disease: a clinical and pathologic study. Am J Dis Child 56(2):344-399

3. Riordan JR, Rommens JM, Kerem B-S et al (1989) Identification of the cystic fibrosis gene: cloning and characterization of complementary DNA. Science. 245(4922):1066-1073

4. Kerem B-S, Rommens JM, Buchanan JA et al (1989) Identification of the cystic fibrosis gene: genetic analysis. Science. 245(4922):1073-1080

5. Frizzell RA, Hanrahan JW (2012) Physiology of epithelial chloride and fluid secretion. Cold Spring Harbor Perspect Med 2(6):a009563

6. Veit G, Avramescu RG, Chiang AN et al (2016) From CFTR biology toward combinatorial pharmacotherapy: expanded classification of cystic fibrosis mutations. Mol Biol Cell 27(3):424-433

7. Bonadia LC, de Lima Marson FA, Ribeiro JD et al (2014) CFTR genotype and clinical outcomes of adult patients carried as cystic fibrosis disease. Gene. 540(2):183-190

8. Ronan NJ, Elborn JS, Plant BJ (2017) Current and emerging comorbidities in cystic fibrosis. Presse Méd 46(6):e125-e138

9. Cutting GR (2015) Cystic fibrosis genetics: from molecular understanding to clinical application. Nat Rev Genet 16(1):45-56

10. Kerem E (2017) Cystic fibrosis: priorities and progress for future therapies. Paediatr Respir Rev 24:14-16

11. Harman K, Dobra R, Davies JC (2018) Disease-modifying drug therapy in CF. Paediatr Respir Rev 26:7-9

12. Rafeeg MM, Murad HAS (2017) Cystic fibrosis: current therapeutic targets and future approaches. J Transl Med 15(1):84

13. Fajac I, Wainwright CE (2017) New treatments targeting the basic defects in cystic fibrosis. Presse Méd 46(6):e165-e175

14. Hurley MN, McKeever TM, Prayle AP, Fogarty AW, Smyth AR (2014) Rate of improvement of CF life expectancy exceeds that of general population —observational death registration study. J Cyst Fibros 13(4):410-415

15. Isa HM, Al-Ali LF, Mohamed AM (2016) Growth assessment and risk factors of malnutrition in children with cystic fibrosis. Saudi Med J 37(3):293-298

16. Scurati-Manzoni E, Fossali EF, Agostoni C et al (2014) Electrolyte abnormalities in cystic fibrosis: systematic review of the literature. Pediatr Nephrol 29(6):1015-1023

17. Giacchi V, Rotolo N, Amato B et al (2015) Heart involvement in children and adults with cystic fibrosis: correlation with pulmonary indexes and inflammation markers. Heart Lung Circ 24(10):1002-1010

18. Akata D, Akhan O, Ozcelik U et al (2002) Hepatobiliary manifestations of cystic fibrosis in children: correlation of $C T$ and US findings. Eur J Radiol 41(1):26-33

19. Sismanlar T, Aslan AT, Kose M et al (2016) Early severe anemia as the first sign of cystic fibrosis. Eur J Pediatr 175(9):1157-1163

20. El-Falaki MM, Shahin WA, El-Basha NR, Ali AA, Mehaney DA, El-Attar MM (2014) Profile of cystic fibrosis in a single referral center in Egypt. J Adv Res 5(5):563-568

21. Mohseni M, Razzaghmanesh M, Parsi Mehr E, Zare H, Beheshtian M, Najmabadi H (2016) Novel CFTR Mutations in Two Iranian Families with Severe Cystic Fibrosis. Iran Biomed J 20(4):201-206

22. Alibakhshi R, Kianishirazi R, Cassiman JJ, Zamani M, Cuppens H (2008) Analysis of the CFTR gene in Iranian cystic fibrosis patients: identification of eight novel mutations. J Cyst Fibros 7(2):102-109

23. Bonyadi M, Omrani O, Rafeey M, Bilan N (2011) Spectrum of CFTR gene mutations in Iranian Azeri Turkish patients with cystic fibrosis. Genet Test Mol Biomarkers 15(1-2):89-92

24. Sahami A, Sadeghifard N, Monsef A, Peyman H (2014) First report of C. 1499G > C mutation in a 6-month-child with cystic fibrosis. Indian J Hum Genet 20(2):199

25. Dooki MRE, Tabaripour R, Rahimi R, Akhavan-Niaki H (2015) Mutation and new polymorphisms insight in introns 11 to 14a of CFTR gene of northern Iranian cystic fibrosis patients. Gene. 564(2):193-196

26. Dooki M-RE, Akhavan-Niaki H, Juibary AG (2011) Detecting common CFTR mutations by reverse dot blot hybridization method in cystic fibrosis first report from Northern Iran. Iran J Pediatr 21(1):51 
27. Zamani RAM (2006) Mutation analysis of CFTR gene in 70 Iranian cystic fibrosis patients. Iran J Allergy Asthma Immunol:3-8

28. Sahami A, Alibakhshi R, Ghadiri K, Sadeghi H (2014) Mutation analysis of exons 10 and 17a of CFTR gene in patients with cystic fibrosis in Kermanshah province, western Iran. J Reprod Infertil 15(1):49

29. Najafi M, Alimadadi H, Rouhani P et al (2015) Genotype-phenotype relationship in Iranian patients with cystic fibrosis. Turk J Gastroenterol 26(3): 241-243

30. Farahmand F, Khalili M, Shahbaznejad L et al (2013) Clinical presentation of cystic fibrosis at the time of diagnosis: a multicenter study in a region without newborn screening. Turk J Gastroenterol 24(6):541-545

31. Farjadian S, Moghtaderi M, Kashef S, Alyasin S, Najib K, Saki F (2013) Clinical and genetic features in patients with cystic fibrosis in southwestern iran. Iran J Pediatr 23(2):212

32. Khatami GR, Mir-Nasseri MM, Seyghali F, Allah-Verdi B, Yourdkhani F (2010) Characteristics of patients with cystic fibrosis: experience in a large referral children's hospital in Tehran, Iran. Middle East J Dig Dis 2(1):20

33. Modaresi MR, Faghinia J, Reisi M, et al (2017) Cystic fibrosis prevalence among a group of high-risk children in the main referral children hospital in Iran. J Educ Health Promot 6:54

34. Naguib ML, Schrijver I, Gardner P et al (2007) Cystic fibrosis detection in high-risk Egyptian children and CFTR mutation analysis. J Cyst Fibros 6(2): 111-116

35. Smith MA, McGarry ME, Ly NP, Zinter MS (2020) Outcomes of children with cystic fibrosis admitted to PICUs. Pediatr Crit Care Med

36. Fallahi GH, Latifi S, Mahmoudi M et al (2016) Growth status and its relationship with serum lipids and albumin in children with cystic fibrosis. Acta Med Iran 54(4):276-279

37. Vahedi L, Jabarpoor-Bonyadi M, Ghojazadeh M, Hazrati H, Rafeey M (2016) Association between outcomes and demographic factors in an Azeri Turkish population with cystic fibrosis: a cross-sectional study in Iran from 2001 through 2014. Iran Red Crescent Med J 18(4):e29615

38. Baghaie N, Kalilzadeh S, Hassanzad M, Parsanejad N, Velayati A (2010) Determination of mortality from cystic fibrosis. Pneumologia 59(3):170-173

39. Kalankesh LR, Dastgiri S, Rafeey M, Rasouli N, Vahedi L (2015) Minimum data set for cystic fibrosis registry: a case study in Iran. Acta Inform Med 23(1):18

\section{Publisher's Note}

Springer Nature remains neutral with regard to jurisdictional claims in published maps and institutional affiliations.

\section{Submit your manuscript to a SpringerOpen ${ }^{\circ}$ journal and benefit from:}

- Convenient online submission

- Rigorous peer review

- Open access: articles freely available online

- High visibility within the field

- Retaining the copyright to your article

Submit your next manuscript at $\boldsymbol{\nabla}$ springeropen.com 